\title{
UN PROYECTO DE APRENDIZAJE-SERVICIO DE ACROSPORT CON PERSONAS CON DIVERSIDAD FUNCIONAL: PERCEPCIONES DE LAS PERSONAS RECEPTORAS DEL SERVICIO
}

\author{
María Teresa Calle-Molina \\ Raquel Aguado-Gómez \\ Ismael Sanz-Arribas \\ $\mathbf{M}^{\mathbf{a}}$ Ángeles López-Rodríguez \\ Universidad Autónoma de Madrid
}

\begin{abstract}
RESUMEN: Este estudio pretende identificar la percepción de las personas receptoras del servicio sobre los efectos de una intervención de acrosport mediante la metodología de Aprendizaje-Servicio. El servicio fue prestado a 62 estudiantes universitarios con discapacidad intelectual por 35 estudiantes del Grado en Ciencias de la Actividad física y el Deporte. Los instrumentos utilizados fueron los diarios reflexivos del alumnado, la grabación de las asambleas celebradas inmediatamente después de finalizar las sesiones y el video-diario que elaboraron al final del semestre. Se realizó un análisis cualitativo con el Software Nvivo-12 Plus y se obtuvieron las siguientes categorías emergentes y resultados: la categoría sobre los aprendizajes adquiridos en la que se referenciaron los contenidos específicos del acrosport y el desarrollo e importancia de habilidades interpersonales e intrapersonales; la categoría sobre dificultades encontradas en la que se hizo alusión a dificultades motrices, atencionales, de seguridad y de relación con los demás. Por último, la categoría sobre los sentimientos en la que se citaban sentimientos generales $y$, también, sentimientos relacionados con sus compañeros y compañeras, con el profesorado y con respecto a sí mismos. En líneas generales, se concluye que las personas que recibieron el servicio percibieron efectos positivos en las categorías emergentes señaladas.
\end{abstract}

PALABRAS CLAVE: aprendizaje-servicio universitario, discapacidad intelectual, acrosport. 


\title{
AN ACROSPORT SERVICE-LEARNING PROJECT WITH PEOPLE WITH FUNCTIONAL DIVERSITY: PERCEPTION OF SERVICE RECEIVERS
}

\begin{abstract}
The objective of this study is to identify the perception of service receivers about the effects of an acrosport intervention through the ServiceLearning methodology. The service was provided to 62 university students with intellectual disability by 35 students of Physical Activity and Sport Science degree. The instruments that were chosen to obtain the information were: the students' reflective journals, the recording of the assemblies that were celebrated just after finishing sessions and the video-diary that they had to elaborate at the end of the semester. A qualitative analysis was performed with the Nvivo-12 Plus software and the following emerged categories and results were obtained: the category on acquired learning in which acrosport contents and the development and importance of interpersonal and intrapersonal skills were referenced; the category on difficulties found related to motor, attention, security and relationship aspects were mentioned; and, finally, the category on feelings in which general feelings and, also, feelings related with classmates, teachers and themselves. In general, we conclude that people who received the service perceived positive effects about the emerged categories pointed.
\end{abstract}

KEYWORDS: University Service-Learning, intellectual disabilities, acrosport.

Recibido: 08/04/2020

Aceptado: 30/06/2020

Correspondencia: María Teresa Calle-Molina, Facultad de Formación de Profesorado y Educación, Universidad Autónoma de Madrid, Ciudad Universitaria de Cantoblanco, 28049 Madrid. Email: mariat.calle@uam.es

\section{INTRODUCCIÓN}

Aunque el Aprendizaje-Servicio (ApS) es definido como "una actividad o programa de servicio solidario protagonizado por los estudiantes, orientado a atender eficazmente las necesidades de una comunidad, y planificada de forma integrada con los contenidos curriculares con el objetivo de optimizar los aprendizajes" (Tapia, 2008, p. 22), es habitual que las publicaciones relacionadas con esta metodología pongan el foco de atención en el análisis y la observación de las competencias que adquiere el alumnado que presta el servicio, y no tanto, sobre los efectos del servicio (Campo, 2015; Carson y Raguse, 2014; Chiva-Bartoll, Ruiz-Montero et al., 2019; Redondo-Corcobado y Fuentes, 2020).

En esta misma línea, algunos autores y autoras consideran que, para valorar la transformación social que se espera del ApS, es necesario evaluar los cambios producidos en el alumnado que presta el servicio, pero también es imprescindible valorar la calidad y la magnitud de los efectos que provocan las intervenciones en la po- 
blación que las recibe (Abdala, 2004; Aramburuzabala, 2013; Cervantes y Meaney, 2013; Campo, 2014; Chiva-Bartoll, Santos, Martínez y Salvador-García, 2019).

Entre estos beneficios, en el contexto de la actividad física, se ha podido comprobar que la población receptora de programas de ApS incrementa su nivel de cooperación, liderazgo, autoconfianza, motivación, inclusión, participación en actividades físicas y grado de satisfacción con el programa (Carson y Raguse, 2014; Cervantes y Meaney, 2013). También se ha comprobado que estas personas adquieren hábitos y estilos de vida saludables (Pérez-Pueyo, Hortigüela-Alcalá, González-Calvo y Fernández-Río, 2019; Vázquez-Toledo, Liesa-Orús y Lozano-Roy, 2017), o incluso, consiguen competencias que les hace disminuir el riesgo de sufrir ahogamientos o accidentes en el agua (Sanz, Calle, Aguado y Garoz, 2019).

Además de lo anterior, la puesta en valor de los efectos del servicio concuerda con la idea de "ciudadanía democrática" que proponen Puig, Martín y Rubio (2017). Según estos autores, este concepto implica una "participación informada, responsable, activa y en colaboración con otros, con el fin de realizar proyectos que no busquen exclusivamente el beneficio privado, sino el bien del conjunto de la sociedad" (p. 124). Junto con esta idea, autores y autoras como An y Decker (2019), Puig y Palos (2006) o Tapia (2008), defienden el ApS como un proyecto articulado, simultáneo, en el que se aprende y se colabora en reciprocidad, y en el que la experiencia y la reflexión son apuestas clave en el desarrollo del proyecto. Esta reciprocidad implica a las personas involucradas tanto en el aprendizaje como en el servicio, atendiendo en ambos casos a los contextos de vida y características de las personas que participan.

Un ejemplo de reciprocidad en el ApS podría estar representado por aquellos proyectos en los que participan personas con discapacidad intelectual (DI). En estos casos, es posible generar una relación simbiótica en la que los estudiantes desarrollan competencias por el hecho de prestar un servicio a personas con DI, y las personas con DI, pueden valorar el servicio y transmitir su opinión sobre el mismo, contribuyendo a su vez a la formación del alumnado.

El hecho de dar voz a las personas con DI que reciben el servicio supone también un reconocimiento social de su contribución a la mejora de dicho Proyecto de ApS (Ríos, 2008), y permite potenciar la idea de que las personas con DI no son una parte de la sociedad, sino sociedad, y reivindicar al mismo tiempo, la necesidad de que desarrollen sus competencias no en espacios privados, sino públicos (Geva y Fish, 2012).

Todo ello, será un paso previo y básico para que dichas personas puedan asumir la responsabilidad del desarrollo de proyectos de ApS para otras personas (Mendía, 2012), e incluso, como señala Tapia (2008), avanzar en una sociedad realmente democrática en sus relaciones, en la que se llegue al concepto de "junto con la comunidad", dejando atrás el de "para la comunidad", que tanto subyace en muchos proyectos de ApS.

Centrándonos en la evaluación de la calidad del servicio, Schalock y Verdugo (2007) cuando abordan el tema de la calidad de vida en los servicios a personas con discapacidad intelectual, señalan como imprescindible recoger información de forma sistemática, analizarla y tomar decisiones. En este sentido, y atendiendo a la implicación de las personas que reciben el servicio en la evaluación, son necesarios 
tanto los procesos de autorregulación en cuanto a su aprendizaje (autoevaluación de qué he aprendido, qué dificultades he tenido, qué errores tengo que mejorar, qué cosas ya domino, cómo me he sentido...), como la recogida de información sobre su percepción de cómo ha influido el proceso de enseñanza en el desarrollo de sus competencias.

Teniendo en cuenta las características de las personas con DI, Docampo y Morán de Castro (2014) señalan que al evaluar tanto las competencias técnicas del saber y saber hacer, como las sociales del saber estar, hay que hacer alguna modificación. Para ello, proponen integrar las dos primeras dimensiones en una sola dimensión llamada "saber haciendo", en la que se evalúa la parte del saber en actividades procedimentales, de forma combinada, sin perder validez, fiabilidad o eficacia. Asimismo, defienden la posibilidad de incorporar apoyos al proceso de evaluación, para facilitar el desarrollo de las actividades de evaluación de conocimientos, habilidades y actitudes.

El acrosport, tiene algunas características que hacen que sea considerado como un contenido con gran potencial educativo (Llamas, Hellín y Moreno, 2004; López, 2010; Reguera y Gutiérrez-Sánchez, 2015; Vernetta, López y Panadero, 1996), no solo por el desarrollo de habilidades motrices como pueden ser el aprendizaje de aspectos técnicos como los agarres, los apoyos, el control postural o los equilibrios, sino porque también hay que sumarle la competencia cognitiva al tener que tomar decisiones, analizar posiciones o buscar soluciones, y el desarrollo de las competencias interpersonales e intrapersonales. Estas últimas juegan un papel muy importante al ser una actividad con carácter cooperativo, participativo e integrador, en la que existe una interdependencia de acción de todas las personas participantes para la consecución del objetivo común, además de la necesidad de asumir distintos roles y responsabilidades durante el desarrollo de las actividades, siendo la ayuda un componente más durante el desarrollo de la actividad.

Algunos estudios que se han centrado en el desarrollo del trabajo cooperativo dentro de la enseñanza de las actividades gimnásticas encontraron mejoras en el autoconcepto del alumnado (Reguera y Gutiérrez-Sánchez, 2015), así como que el alumnado percibe una mejora en sus competencias afectivo-sociales como el trabajo en equipo y en las competencias cognitivas como la toma de decisiones o la aportación de ideas (Ávalos, Martínez y Merma-Molina, 2019).

Las características señaladas hacen que el contenido de acrosport se convierta en un contenido propicio para las clases de educación física independientemente de si las personas que asisten tienen discapacidad intelectual o no, aunque dependiendo del enfoque metodológico llevado a cabo por el profesorado se desarrollarán unas competencias más que otras.

En virtud de lo planteado con anterioridad, este trabajo plantea el siguiente objetivo: identificar la percepción de las personas receptoras del servicio sobre los efectos de una intervención de acrosport a través de la metodología de ApS. 


\section{Metodología}

Se ha llevado a cabo una investigación de tipo cualitativo. A continuación, se presentan las características de los participantes, los recursos materiales y humanos, la descripción del proyecto ApS, así como las técnicas, instrumentos y procedimientos utilizados en la recogida de información.

\subsection{Características de los participantes}

En esta investigación han participado 62 personas (31 hombres y 31 mujeres) matriculadas en la asignatura "Desarrollo de conductas motrices y su relación con el ámbito deportivo" perteneciente al Título Propio de Formación para la Inclusión Laboral del Programa Promentor-UAM. Todas ellas son mayores de 18 años y tienen discapacidad intelectual leve o moderada y, en dos de los casos, una discapacidad física moderada. En todos los casos disponen de un alto grado de autonomía funcional y/o de los apoyos personales y técnicos necesarios para desarrollar las tareas requeridas. Dicho esto, se ha garantizado el anonimato de todas las personas que han participado en este estudio y se han respetado todas las normas éticas de investigación nacionales e internacionales.

\subsection{Recursos materiales y humanos}

2.2.1. Los recursos materiales necesarios para realizar esta investigación fueron:

- Una videocámara para la grabación de las sesiones.

- Un micrófono inalámbrico.

- Una sala con pavimento acolchado para atenuar el impacto de caídas.

- Cuadernos para recoger la información de los diarios reflexivos del alumnado.

2.2.2. Los recursos humanos que han intervenido en el proceso del presente estudio fueron:

- La docente de la materia del Grado universitario.

- 35 estudiantes (19 hombres y 16 mujeres) matriculados en la materia desde el curso 2017-2018 hasta el curso 2019-2020 incluido, que participaron voluntariamente en un servicio directo.

\subsection{Descripción del Proyecto ApS}

Las necesidades que se pretenden cubrir en el colectivo receptor del servicio están relacionadas con la adquisición de hábitos de práctica de actividad física saludable y la toma de conciencia de la importancia de tener una vida activa y de calidad.

Para dar respuesta a las necesidades indicadas, desde la materia "Fundamentos de los deportes individuales y su didáctica II: Gimnasia" perteneciente al 
Grado de Ciencias de la Actividad Física y del Deporte, se ofreció al alumnado matriculado la posibilidad de participar en el proyecto ApS descrito. Esta opción se encuentra dentro de los trabajos habitualmente ofrecidos siendo, por tanto, parte evaluable de la asignatura. El alumnado participante en este proyecto tuvo la posibilidad de adquirir, experimentar y perfeccionar su capacidad docente, diseñando e implementando acciones prácticas relacionadas con el acrosport, dirigidas a personas con diversidad funcional, valorando las posibilidades de generar propuestas físico-deportivas en diferentes contextos, con carácter inclusivoeducativo-recreativo y adaptado a las capacidades y condiciones de las personas participantes.

Antes de ofrecerles esta propuesta, el alumnado tuvo la oportunidad de participar en una asamblea grupal al inicio del curso con el alumnado de Promentor. Esta asamblea estaba diseñada para que ambas partes se conociesen y relacionasen. Posteriormente, el alumnado del grado que decidió voluntariamente participar en el proyecto se reunió con uno de los profesionales del equipo de Promentor-UAM. En esta reunión se habló sobre las características particulares de las personas receptoras del servicio y sobre las medidas de seguridad y prevención de accidentes que debían ser adoptadas.

A continuación, el alumnado de grado se reunió con la docente de su asignatura y tutora durante el desarrollo del ApS para visualizar vídeos. En estas grabaciones aparecía el colectivo al que posteriormente se iban a dirigir efectuando experiencias deportivas previas. El objetivo de esta tarea era analizar las características del alumnado y las posibles adaptaciones que deberían tenerse en cuenta en el diseño y desarrollo de su propuesta.

Posteriormente, el alumnado mantuvo reuniones periódicas con su tutora para abordar las cuestiones que iban surgiendo en el diseño y desarrollo de las sesiones y que consideraban que requerían adaptaciones. La tutora de la materia supervisó de manera presencial el desarrollo de todas las sesiones.

El contenido implementado durante las sesiones fue el acrosport, tratando de desarrollar tanto competencias motrices como cognitivas, interpersonales e intrapersonales, contribuyendo de esta forma al desarrollo integral de las personas.

Las sesiones comenzaban con una explicación de los objetivos y recordatorio de lo trabajado en sesiones anteriores. Durante el desarrollo de éstas se incidía en que las personas receptoras del servicio conociesen el por qué y para qué de cada actividad, en que observasen, analizasen posiciones o figuras y diesen feedback a los compañeros o compañeras de clase para intentar mejorar, terminando las sesiones con una reflexión grupal.

Finalmente, al término del curso, el alumnado de grado y del título propio volvía a reencontrarse en una fiesta inclusiva, en la que realizaban algunas actividades lúdicas en un espacio abierto del Campus Universitario. 


\subsection{Técnicas, instrumentos y procedimiento utilizados en la recogida de información}

Para la consecución de los objetivos planteados, se han utilizado técnicas e instrumentos narrativos, en los que el alumnado con DI ha podido reflexionar sobre lo que ocurrió en la sesión en tres momentos diferentes: en la reflexión final de la sesión, en el diario reflexivo realizado horas después de finalizar la sesión y, por último, en el montaje de un video-diario dos meses después de haber recibido todas las sesiones de acrosport.

El alumnado poseía características similares en cuanto a su edad y tipo de discapacidad, sin embargo, existía diversidad respecto a sus capacidades y recursos expresivos. Por ello, las técnicas narrativas utilizadas fueron orales y escritas, con el fin de ofrecer diversas opciones que facilitaran su comunicación.

A continuación, se explican detalladamente las técnicas e instrumentos utilizados:

- Observación no participante de la sesión en la que se recogen imágenes y sonido del desarrollo de ésta con una videocámara. Para esta investigación se utilizaron las reflexiones finales de cada sesión en las que se ofrecía al alumnado de Promentor la posibilidad de expresar qué había ocurrido en la sesión y cómo se había sentido. Estas asambleas eran moderadas por el alumnado de Grado que había impartido la sesión.

- Diario reflexivo: el alumnado de Promentor debía realizar una reflexión el mismo día que recibía la práctica. Este diario era semiestructurado con el fin de guiar la reflexión en torno a sus aprendizajes adquiridos y los sentimientos que habían experimentado durante la sesión. Este diario era recogido por el alumnado de Grado el día siguiente a la práctica. Se valoraba y se proporcionaba el feedback correspondiente $y$, al mismo tiempo, se obtenía información para el diseño de la siguiente práctica. Además, la decisión de utilizar los diarios reflexivos permitía que el alumnado de Promentor pudiera reflexionar con cierta perspectiva y de manera individual sobre lo que había ocurrido en la sesión ese mismo día. Se trataba así de obtener información sobre lo que realmente para el alumnado había sido significativo de la sesión, compensando a veces un exceso en la conducción de las reflexiones al final de la sesión, por parte del alumnado de Grado.

- Vídeo-diario: con el alumnado de Promentor se acordó la realización del montaje de un vídeo sobre uno de los contenidos que habían trabajado durante el semestre. Uno de los grupos de cada curso participante realizó el vídeo sobre el contenido de acrosport. Se trataba de recoger los siguientes aspectos: información sobre el lugar, profesorado, contenido y fechas en las que se desarrolló la práctica, una breve descripción de las sesiones y los contenidos más significativos y, por último, qué habían aprendido y cómo se habían sentido. Este recurso tenía como objetivo que los integrantes del grupo revisaran sus diarios reflexivos, volvieran a recordar el contenido dos meses después de haberlo practicado y fueran capaces de contar de manera breve qué hicieron, 
qué aprendieron y cómo se sintieron. La información obtenida a partir de este recurso nos ofreció otra perspectiva temporal del alumnado sobre el proceso de enseñanza y aprendizaje desarrollado.

\section{Resultados Y DISCUSIÓN}

La atención de esta investigación se centra en los receptores del servicio. Tratando de valorar a través de la percepción del alumnado receptor del servicio, si el contenido de acrosport a través de la metodología de aprendizaje-servicio ha conseguido y en qué medida desarrollar sus competencias. Para ello, se parte de las preguntas: ¿qué he aprendido? y ¿cómo me he sentido?

Se ha llevado un proceso de análisis cualitativo, de carácter inductivo utilizando el Software Nvivo 12 Plus para el análisis de la información de los diarios reflexivos (Diario), las reflexiones al finalizar las sesiones (Reflex) y los video diarios (VideoD) realizados al finalizar el semestre.

Se comenzó con una codificación abierta y, posteriormente, con una codificación axial de la que finalmente surgieron tres categorías principales (Figura 1): 1) Aprendizajes adquiridos; 2) Dificultades encontradas; 3) Sentimientos.

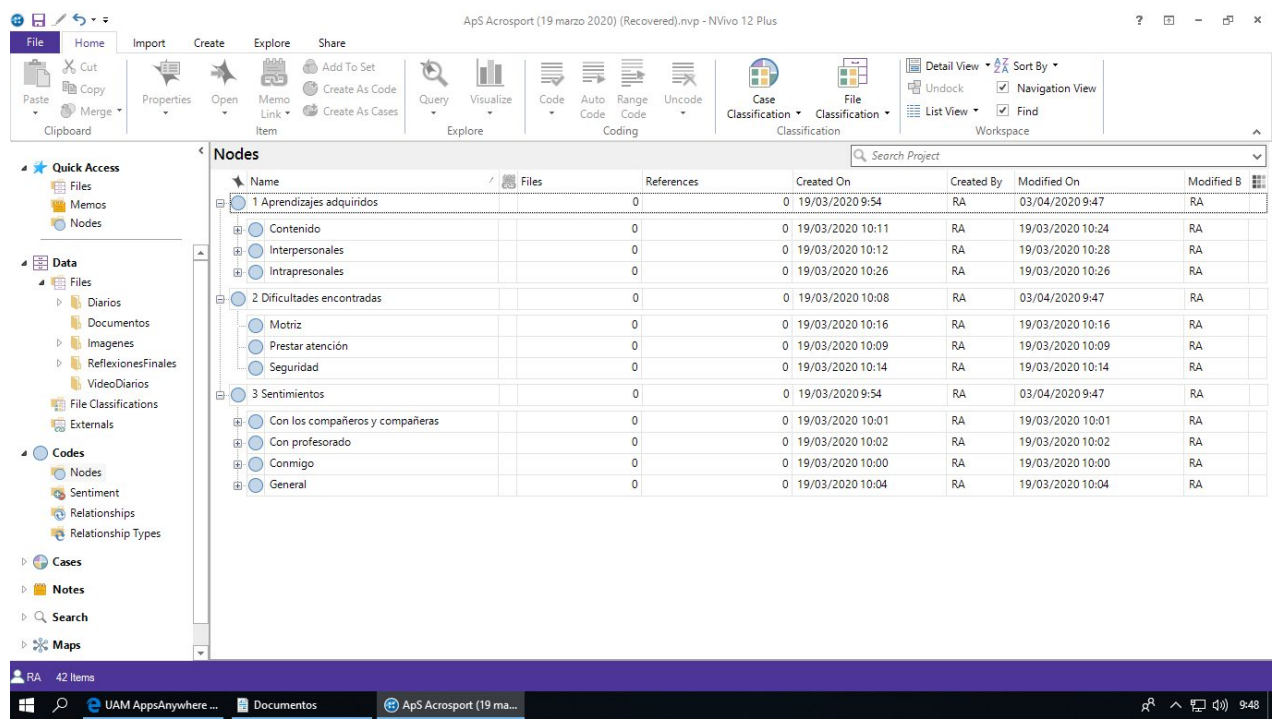

Figura 1. Categorías principales

La categoría Aprendizajes adquiridos asociada más directamente al desarrollo de su competencia cognitiva y motriz, a través del "saber haciendo", hace referencia a la percepción que tiene el alumnado con DI acerca de los aprendizajes adquiridos en las sesiones de acrosport. En esta categoría hemos encontrado referencias relacionadas con tres aspectos: Ios contenidos propios de esta disciplina, los aprendizajes interpersonales y los aprendizajes intrapersonales. 
Respecto a la percepción sobre su aprendizaje con relación a los contenidos de la disciplina de acrosport hemos encontrado referencias acerca de diversos contenidos. Los que más señala el alumnado son los relacionados con la formación de figuras y pirámides, los distintos roles que pueden adoptar en la formación de éstas y el trabajo del equilibrio:

He aprendido a hacer diferentes formas en grupo y me lo pasé muy bien con todos (Diario_JG_15-11-2017)

... hacer posturas en grupos de cuatro personas haciendo de portor y ágil (Diario_ ARP_27-11-2019)

Me han servido para mejorar el equilibrio (VideoD_2017-2018_P2 [2:25,1 - 2:32,8])

Sin olvidar como puntos importantes la adopción de apoyos y posturas corporales adecuadas en la realización de las actividades propuestas:

He aprendido a poner las manos bien en el suelo para hacer las figuras (Diario_ELT_1912-18)

Los apoyos... tienen que ser equilibrados (Reflex_15-11-2017a [2:00,8 - 2:11,4])

Estar a cuatro patas... con brazos rectos, espalda recta y cabeza (Reflex_15-11-2017b $[1: 28,1-1: 36,3])$

También están presentes en los contenidos que identifican que han aprendido aspectos relacionados con la realización de distintos tipos de contrapesos y los tipos de agarres:

Empezamos haciendo pinzas con otros compañeros, nos cogíamos con los dedos de las manos y con los brazos estirados tirábamos. Después igual, pero agarrando por las muñecas y también con los brazos cruzados y por último de espalda a nuestro compañero y agarrados de las manos tirábamos hacia nosotros (Diario_PM_06-11-2019)

... agarres como: $1^{\circ}$ mano-mano; mano-antebrazo; antebrazo y de pinza (Diario_ ARR_19-12-18)

Hicimos los contrapesos, el contrapeso interno y el contrapeso externo (Diario_ ARP_27-11-2019)

En estos testimonios se puede observar que el alumnado identifica los aspectos motrices y cognitivos básicos que se trabajaron en las sesiones, tanto al finalizar las mismas como transcurrido un tiempo desde la intervención, demostrando una consolidación en el aprendizaje específico del contenido, coincidiendo con Ávalos et al. (2019).

Otro de los aspectos destacables dentro de esta categoría son las cuestiones relacionadas con la seguridad para llevar a cabo las actividades de esta disciplina, debido al riesgo que puede suponer no tener presentes y claras algunas cuestiones mínimas de seguridad (León, 2004) y la utilización de las ayudas:

... actividad era con la seguridad de los compañeros que nos ayudaron hacer figuras de cuatro y cinco grupos (Diario_JP_20-11-2019)

La colocación de una persona por encima de otra para ello es muy importante lo pri- 
mero cargar bien el peso... no en la zona de la espalda sino en la zona del culete para evitar daños. Y luego también es muy importante hacerlo con cuidado y despacio ( $\mathrm{Vi}$ deoD_2018-2019_P1 [0:09,8 - 0:32,1])

Estos resultados coinciden con los presentados por Sanz et al. (2019), en los que se afirma que la metodología ApS es una herramienta útil para mejorar el nivel de seguridad de las personas con DI.

Por otro lado, en cuanto al desarrollo de competencias relacionadas con la interacción necesaria para llevar a cabo el contenido concreto de acrosport, y considerando las características específicas del mismo que ya comentamos en la introducción, las referencias encontradas a este respecto destacan la importancia del trabajo en equipo y de la cooperación:

Me resultó super importante porque es una manera de trabajar en equipo ( $\mathrm{Vi}$ deoD_2018-2019_P1 [0:47,6 - 0:55,8])

Se utiliza mucho el trabajo en equipo, la cooperación y la ayuda entre compañeros (Diario_GG_8-11-2017)

También la importancia de la ayuda entre compañeros, y el relacionarse con todas las personas de clase:

Antes no lo hemos podido hacer solos y al estar con JM lo hemos intentado otra vez, muchas veces y nos hemos podido levantar... le he dicho ¡empuja!... y nos hemos podido levantar los dos (Reflex_15-11-2017a [1:17,1 - 1:33,0])

... he aprendido a jugar en equipo con todos los chicos y chicas y con los profesores a la hora de jugar (Diario_CM_12-12-19)

Como podemos ver, el trabajo en equipo y la cooperación, aspectos tan característicos del contenido de acrosport, no han sido pasados por alto por el alumnado y son identificados como algo importante dentro de este contenido coincidiendo con lo indicado por diversos autores (Llamas et al., 2004; López, 2010; Reguera y Gutiérrez-Sánchez, 2015; Vernetta et al., 1996).

Y, además, destacaban la importancia no solo de su responsabilidad y competencias propias, sino de tener en cuenta las capacidades de los demás, para poder llevar a cabo las actividades propuestas de forma grupal, así como confiar en los demás miembros del grupo:

Me ha gustado mucho la clase porque puedes observar las diferentes capacidades motrices de mis compañeros y de mí y darles el apoyo que necesiten en cada momento y la confianza que hay entre los distintos compañeros (Diario_GG_15-11-2017)

He aprendido a confiar más en mis compañeros (Diario_ARR_19-12-18)

En estas afirmaciones se pone de manifiesto que el alumnado percibe que la confianza, la cooperación y la socialización con el grupo se ha visto incrementada tras la recepción del servicio. Estos resultados coinciden con los planteados por ChivaBartoll, Santos et al. (2019), que afirman que los receptores del servicio deben ser una prioridad de los programas de ApS. 
Por último, dentro de esta categoría encontramos reseñas sobre el desarrollo de las competencias intrapersonales, las cuales hacen referencia al conocimiento de uno mismo a partir de la participación en las sesiones de acrosport.

Se trata de coger confianza mediante juegos y actividades (Diario_ARR_5-12-18)

La confianza es importante para tener la seguridad en uno mismo (Diario_AR_8-11-17)

A mí me ha servido para mejorar la timidez y así un poco la confianza, y he perdido mucho la vergüenza (VideoD_2017-2018_P2 [2:32,7 - 2:38,4])

La bibliografía revisada y los comentarios expuestos anteriormente indican que se produce un avance en la percepción de la confianza en uno mismo, en el conocimiento de las propias capacidades y en la disminución del sentimiento de vergüenza, con lo que esto significa para la mejora de la calidad de vida de las personas (Carson y Raguse, 2014; Cervantes y Meaney, 2013; Schalock y Verdugo, 2007)

La segunda categoría, Dificultades encontradas, hace referencia a la percepción del alumnado con DI sobre las dificultades que han tenido en las sesiones de acrosport. En esta categoría principalmente hemos encontrado referencias acerca de las dificultades motrices, las dificultades relacionadas con cuestiones de seguridad, la dificultad atencional y las dificultades con respecto a establecer relaciones con los demás.

Las referencias sobre la percepción acerca de las dificultades motrices apuntaban a contenidos propios de acrosport o a la diferencia sobre la percepción previa de la dificultad de la actividad y las dificultades encontradas una vez que lo realizaban de forma práctica:

... teníamos que hacer parejas con el número de apoyos que hacer, haciendo también formas de equilibrio: agarrándose del uno al otro estando enfrente, de lateral (de lado), sentados, de espaldas intentando levantarnos de uno al otro: JM y yo nos costó estando espalda a espalda agarrados de los brazos y levantarnos empujando la espalda al otro pero intentando una y otra vez conseguimos (Diario_NT_15-11-2017)

Lo más complicado el agarre en pinza [...] depende del peso de cada uno (Reflex_9-1-2019 [0:18,3 - 0:27,1])

He aprendido que te lo puedes pasar bien haciendo deporte y que a veces es más difícil de lo que parece hacer una actividad deportiva (Diario_HC_12-12-18)

Estos comentarios demuestran que el alumnado percibe la sesión de acrosport como un reto que deben superar, pero que, al mismo tiempo les permite disfrutar y pasarlo bien. Por lo tanto, es muy posible que estas personas se sientan motivadas a repetir la actividad y a adquirir el hábito de realizar actividad física (Pérez-Pueyo et al., 2019; Vázquez-Toledo et al., 2017).

En relación con las dificultades asociadas con cuestiones de seguridad, se mencionaban algunas actividades que tenían como objetivo el aumento de la confianza entre los compañeros y que, en algunos casos, habían supuesto la percepción de falta de seguridad o señalaban que se habían hecho daño: 
Hoy he aprendido a andar con los ojos tapados, yo iba muy despacio porque tenía miedo de tropezar con algún compañero y caerme (Diario_PM_06-11-2019) A mí me da miedo echarme para atrás (Reflex_19-12-2018 [5:05,8 - 5:10,9]) Yo cambiaría lo de la figura del portor para no hacerme daño en la espalda (Reflex_12-11-2019 [5:08,6 - 5:13,2])

Estos resultados demuestran que el alumnado es consciente de sus propias limitaciones y de que puede sufrir lesiones si no adopta las medidas de seguridad y prevención adecuadas. Coincidiendo con los resultados de investigaciones previas que se indican que los proyectos de ApS pueden mejorar la seguridad de los receptores (Sanz et al., 2019).

En cuanto a las categorías relacionadas con la percepción sobre la dificultad atencional y las dificultades con respecto a establecer relaciones con las demás personas, suponían un reto fundamental para un grupo muy reducido de alumnos, teniendo en cuenta la naturaleza del contenido con respecto a la utilización de espacios personales cercanos:

... me ha parecido genial y divertido lo que me cuesta mucho es relacionarme con los compañeros, pero me relaciono muy bien (Diario_JMM_12-12-18)

... me ha pareció genial y me ha gustado mucho. Lo que más me cuesta es estar atento estar junto los compañeros (Diario_JMM_5-12-18)

Por último, la categoría Sentimientos hace alusión a la percepción del alumnado con DI acerca de sus propios sentimientos durante las sesiones. En esta categoría hemos encontrado referencias acerca de la percepción sobre los sentimientos que habían experimentado de manera general, sobre sus propios sentimientos, los sentimientos en cuanto a su relación con los compañeros y, por último, en cuanto a la relación con el profesorado.

En la primera subcategoría relacionada con la percepción sobre los sentimientos que consideraban que habían experimentado de manera general durante las sesiones de acrosport, encontramos referencias sobre: alegría, cansancio, diversión, felicidad, tranquilidad y calma.

Con relación a los sentimientos de alegría uno de los testimonios hacía referencia a la última de las clases que tendrían de acrosport de la siguiente manera:

$\mathrm{S}, \mathrm{N}$ y $\mathrm{S}$ nos hicieron un reportaje a nosotros por los momentos que hemos pasado juntos por estos dos cursos emocionantes, alegres y maravillosas que algunos nos pusimos a llorar por la emoción que teníamos dentro de nosotros y eso para nosotros nos pusimos tristes (Diario_CM_27-11-2019)

Nos hemos sentido... divertido... alegres y fue interesante (VideoD_2019-2020_P1c> $[0: 48,5-0: 59,6])$

La mayor parte de referencias en esta categoría mencionaban un sentimiento de diversión. Se presentan algunas de ellas que, además, mencionaban el aprendizaje o el trabajo en equipo como componentes secundarios de la diversión: 
Me he sentido genial ha sido divertido con JG, AM y CC en grupo y me gustaría repetir otra vez (Diario_AR_8-11-17)

La clase me ha parecido muy amena y novedosa por las técnicas del portor y el ágil que no las conocía. Ha estado divertida y he aprendido mucho estos días (Diario_ARR_1912-18)

Me ha parecido divertida y muy activa (Reflex_5-12-2018b [2:50,4 - 3:00,9])

Otras citas, aunque aludían a sentimientos de diversión, también apuntaban a un sentimiento de cansancio percibido tras terminar la sesión:

...me ha gustado la actividad, es divertido, aunque un poco cansado (Diario_ELT_0611-2019)

Otros testimonios apuntaban al sentimiento de felicidad en general, aunque mencionaban otros sentimientos que asociaban con ese estado:

Hemos acabado la clase, me he sentido bien, a gusto, feliz y he aprendido a hacer figuras con ayuda de mis compañeros (Diario_PM_20-11-2019)

Por último, algunos testimonios recogidos mencionaban la calma y la tranquilidad como sentimientos percibidos en las sesiones de acrosport:

Me lo he pasado muy bien y me siento tranquilo (Diario_NT_15-11-2017)

A mi este deporte me gusta porque es una forma para estirar lo bien lo que es la parte lumbar y hace que te sientas mejor, tranquilo (VideoD_2018-2019_P1 [3:45,7 - 3:59,4])

Con relación a sus percepciones sobre los sentimientos que se manifestaban con respecto a ellos mismos, encontramos referencias en los testimonios que apuntaban a la confianza en uno mismo. En el caso que se muestra como ejemplo en primer lugar aludiendo a la reflexión de las acciones motrices:

Me siento fenomenal y se nota en mí que me acuerdo de todo, no siempre, pero hay que tener la confianza y la conciencia de saber lo que hacemos (Diario_AR_15-11-17) ción:

Por otro lado, se encontraron numerosas referencias que apuntaban a la supera-

Ha sido una experiencia muy buena, muy positiva e ir desarrollando todas las sesiones y con buenas capacidades en el entorno de educación física y el deporte y seguir superando los retos deportivos de día a día (Diario_AR_22-11-17)

He aprendido a hacer diferentes agarres de manos, a hacer unos ejercicios de espalda superando el miedo y las diferentes figuras de acrosport que se pueden hacer (Diario_ARP_19-12-18)

A mí me ha servido para mejorar la timidez y así un poco la confianza, y he perdido mucho la vergüenza (VideoD_2017-2018_P2 [2:32,7 - 2:38,4])

Estas afirmaciones están en línea con algunas investigaciones como la llevada a cabo por Reguera y Gutiérrez-Sánchez (2015). En ésta, después de llevar a cabo un proceso de enseñanza de acrosport, se comprobó que los niveles de 
autoconcepto mejoraban, principalmente en estudiantes con problemas físicos y/o emocionales.

Puesto que nunca se habló de extenuación o fatiga excesiva, todos los sentimientos encontrados en esta categoría se relacionan con sensaciones positivas y placenteras. La presencia de estas percepciones entre la población receptora de proyectos de ApS es una de las metas que deben alcanzar los servicios de calidad. La bibliografía demuestra que cuando la actividad física genera sensaciones positivas, es muy posible que se mejore la adherencia al ejercicio físico, y por tanto, se instauren hábitos y estilos de vida saludables que mejoren la calidad de vida (Pérez-Pueyo et al., 2019; Vázquez-Toledo et al., 2017).

En cuanto a la percepción que tuvieron sobre sus sentimientos respecto a las relaciones que se establecieron con los compañeros y compañeras, la mayor parte de referencias encontradas hacen alusión a aspectos positivos y, algunas mínimas, mencionaban comportamientos disruptivos que tenían que ver con los momentos posteriores al desarrollo de las tareas. Sobre los aspectos positivos que se producían durante la sesión se mencionaba el acogimiento, el agradecimiento y la confianza en los demás.

Las referencias en cuanto a las relaciones establecidas con los compañeros y compañeras señalaban el acogimiento, incluso dándole una perspectiva de respeto de las diferentes capacidades de los compañeros con respecto al contenido propuesto y a la importancia de las ayudas para generar el mismo:

Pues he aprendido a hacer otros juegos. También a estar con los compañeros y pasarlo bien. Me ha gustado estar con todos en clase de EF. He estado genial con todos mis compañeros es lo más importante (Diario_PM_06-11-2019)

Me he sentido bien con mis compañeros haciendo un trabajo en equipo y viendo las diferentes capacidades de cada uno y así poder ayudarles en cada momento (Diario_GG_22-11-2017)

La mejora de la relación social del grupo es uno de los aspectos que el alumnado también valora en otras investigaciones relacionadas con el contenido de acrosport (Ávalos et al., 2019).

También se hacía referencia al agradecimiento y a la confianza en los compañeros y compañeras en los diarios y en las reflexiones grupales que se desarrollaban al término de las sesiones:

A mí me parece que hemos estado mucho no solo en el trabajo en equipo, si no la confianza entre las personas (Reflex_19-12-2018 [7:41,2 - 7:58,6])

Lo que más me ha gustado de esta primera sesión es cuando estábamos en parejas y una tenía que llevar el antifaz puesto y el otro compañero guiaba, así todo el rato (Diario_ARP_06-11-2019)

En cuanto a su percepción acerca de sus sentimientos en relación con el profesorado, es decir, el alumnado de Grado que realizaba la intervención de ApS, encontramos referencias que manifiestan alegría por conocerlos, agradecimiento y 
reconocimiento. Otras investigaciones previas, relacionadas con los sentimientos de los receptores del servicio a partir de metodologías ApS, también recogían información en esta misma línea (Ríos, 2008). Las referencias que apuntaban a la alegría por conocerlos, en algunas ocasiones también mostraban agradecimiento:

Los profesores nos han dado de nuestras clases y me ha gustado mucho. Luego hemos tenido tutoría para preparar el vídeo. Me ha gustado mucho conocer a los chicos de grado, muchas gracias por quedarnos en estas sesiones (Diario_FG_27-11-2019)

En aquellas referencias que se hace alusión al sentimiento de agradecimiento hacia el alumnado de Grado que dio el servicio, también existen ciertos matices que señalan el reconocimiento hacia su labor docente. Encontramos numerosas citas en los tres años analizados que apuntaban a su profesionalidad, paciencia y esfuerzo e incluso se manifestaba el deseo de volver a trabajar con ellos:

Lo que me ha gustado en general es como han explicado S, N y S las actividades. Gracias a ellos hemos aprendido a hacer las figuras nuevas que antes no sabíamos hacer (Diario_ARP_27-11-2019)

Me lo he pasado bien. Es una lástima que acabe y espero volver a trabajar con ellos (Diario_HC_27-11-2019)

Felicito a I que fue muy paciente y la clase la preparó genial (Diario_ARR_5-12-18)

Como puede observarse y al igual que ocurría en investigaciones previas que han sido presentadas en el marco teórico, los comentarios recogidos en este estudio muestran un alto grado de satisfacción con el servicio y con el profesorado que lo impartió (Carson y Raguse, 2014; Cervantes y Meaney, 2013).

\section{Conclusiones}

Los resultados obtenidos en este trabajo indican que el alumnado con DI participante en esta investigación tiene la percepción de adquirir aprendizajes técnicos, tanto cognitivos como motrices relacionados con los contenidos de acrosport, cuando éstos son impartidos por el alumnado universitario a través de la metodología ApS. Otros aprendizajes señalados están relacionados con las relaciones interpersonales, el trabajo en equipo, la ayuda o la aceptación de las capacidades de los demás como elementos que han surgido de la práctica de acrosport. Asimismo, también han existido aprendizajes intrapersonales relacionados con la concentración, la confianza o el conocimiento de las propias capacidades como aprendizajes adquiridos a partir de estas intervenciones.

Podemos concluir que a partir de estas intervenciones el alumnado con DI participante también fue capaz de reflexionar acerca de las dificultades encontradas en esta práctica, resaltando aspectos motrices, atencionales o relacionados con la seguridad como elementos presentes en estas intervenciones.

Por último, el alumnado participante en esta investigación reflexionó acerca de su percepción con respecto a sus sentimientos y, como se muestra en los resultados de la investigación, se puede concluir lo siguiente: el alumnado con DI experimentó y 
expresó múltiples sentimientos relacionados con la alegría, la diversión y la felicidad y otros, no tan numerosos, como el cansancio o la tranquilidad y la calma derivados de su práctica. Respecto a los sentimientos sobre sí mismos se aludió al aumento de la superación y la confianza en sí mismos. Además, éstos expusieron su percepción acerca de sentimientos como el acogimiento, el aumento de la confianza en los demás o las muestras de agradecimiento hacia sus compañeros. Por último, se aludió a sentimientos de agradecimiento y reconocimiento y se mostró alegría por tener la posibilidad de conocer al alumnado de los dos grados universitarios citados.

\section{AgradeCimientos}

En este apartado se quiere agradecer la inestimable colaboración del Programa Promentor de la Universidad Autónoma de Madrid para el desarrollo de esta investigación.

\section{RefERENCIAS BIBLIOGRÁFICAS}

Abdala, E. (2004). Manual para la evaluación del impacto en programas de formación para jóvenes. Montevideo: CINTERFOR.

An, J. y Decker, J. T. (2019). Utilizing a critical service-learning approach in an adapted physical education course. Journal of Physical Education, Recreation \& Dance, 90(4), 7-14. https://doi.org/10.1080/07303084.2019.1568932

Aramburuzabala, P. (2013). Aprendizaje-Servicio: Una herramienta para educar desde y para la justicia social. Revista Internacional de Educación para la Justicia Social, 2(2), 5-11. http://dx.doi.org/10.15366/riejs2013.2.2

Ávalos, M. A., Martínez, M. A. y Merma-Molina, G. (2019). Implementation and Evaluation of a Collaborative Gymnastic Strategy. Revista Internacional de Medicina y Ciencias de la Actividad Física y el Deporte, 19(76), 579-598. http://dx.doi. org/10.15366/rimcafd2019.76.001

Campo, L. (2014). Aprendizaje servicio y educación superior. Una rúbrica para evaluar la calidad de proyectos. Tesis doctoral. Universitat de Barcelona, Barcelona. Recuperado de https://www.uv.es/aps/doc/Tesis/Tesis\%20Campo\%20Cano,\%20 Laura.pdf

Campo, L. (2015). Evaluar para mejorar los proyectos de aprendizaje servicio en la universidad. RIDAS, Revista Iberoamericana de Aprendizaje y Servicio, 1, 91-111. https://doi.org/10.1344/ridas2015.1.6

Carson, R. L. y Raguse, A. L. (2014). Systematic Review of Service-Learning in Youth Physical Activity Settings. Quest, 66(1), 57-95. https://doi.org/10.1080/00336297 .2013 .814578

Cervantes, C. M. y Meaney, K. S. (2013). Examining Service-Learning Literature in Physical Education Teacher Education: Recommendations for Practice and Research. Quest, 65(3), 332-353. https://doi.org/10.1080/00336297.2013.773533 
Chiva-Bartoll, O., Ruiz-Montero, P., Martín, R., Pérez, I., Giles, J., García-Suárez, J. y Rivera-García, E. (2019). University Service-Learning in Physical Education and Sport Sciences: A systematic review. Revista Complutense De Educación, 30(4), 1147-1164. https://doi.org/10.5209/rced.60191

Chiva-Bartoll, O., Santos, M. L., Martínez, F. y Salvador-García, C. (2019). Valoración del impacto del aprendizaje-servicio universitario en el ámbito de la actividad física y el deporte: una propuesta desde la teoría de stakeholders. Publicaciones, 49(4), 29-48. https://doi.org/10.30827/publicaciones.v49i4.11727

Docampo, G. y Morán de Castro, M. C. (2014). Evaluación de la competencia profesional en personas con discapacidad intelectual. Una propuesta de adaptación de la metodología e instrumentos INCUAL para la igualdad de oportunidades. Revista Española de Discapacidad, 2(1), 71-96. http://doi.org/10.5569/23405104.02.01.04

Geva, E. y Fish, T. (2012). Cómo los proyectos de Aprendizaje Servicio pueden favorecer la participación social en personas con discapacidad intelectual. En M. Verdugo, F. Jordán de Urríes, D. Velázquez, T. Nieto y M. Crespo (Eds.), VIII Jornadas Científicas Internacionales de Investigación sobre Personas con Discapacidad. Cambio organizacional y apoyo a las graves afectaciones. Dos prioridades (pp. 1-9). Salamanca, España: Instituto Universitario de integración en la comunidad.

Llamas, L., Hellín, G. y Moreno, J. A. (2004). La enseñanza de habilidades gimnásticas y acrobáticas en la etapa escolar. Revista Internacional de Medicina y Ciencias de la Actividad Física y el Deporte, 4(14), 82-105. Recuperado de http://cdeporte. rediris.es/revista/revista14/arthabgimnasticas.htm

López, R. (2010). El acrosport: una propuesta cooperativa para el desarrollo. EmásF: revista digital de educación física, (4), 37-52. Recuperado de https://emasf.webcindario.com/El_acrosport_una_propuesta_cooperativa.pdf

León, J. A. (2004). Seguridad e higiene en la práctica saludable del acrosport. Revista Internacional de Medicina y Ciencias de la Actividad Física y el Deporte, 4(16), 276-285. Recuperado de http://cdeporte.rediris.es/revista/revista16/artseguridad. htm

Mendía, R. (2012). El Aprendizaje-Servicio como una estrategia inclusiva para superar las barreras al aprendizaje y a la participación. Revista Educación Inclusiva 5(1), 71-82. Recuperado de https://www.revistaeducacioninclusiva.es/index.php/ $\mathrm{REl} /$ article/view/222

Pérez-Pueyo, A., Hortigüela-Alcalá, D., González-Calvo, G. y Fernández-Río, J. (2019). Muévete conmigo, un proyecto de aprendizaje servicio en el contexto de la educación física, la actividad física y el deporte. Publicaciones, 49(4), 183-198. https://doi.org/10.30827/publicaciones.v49i4.11735

Puig, J. M. y Palos, J. (2006). Rasgos pedagógicos del Aprendizaje-servicio. Cuadernos de Pedagogía, 357, 60-63. Recuperado de https:/dialnet.unirioja.es/ ejemplar/132365 
Puig, J., Martín, X. y Rubio, L. (2017). ¿Cómo evaluar proyectos de aprendizaje servicio? Voces de la Educación, 2(4), 122-132. Recuperado de https://www.revista. vocesdelaeducacion.com.mx/index.php/voces/article/view/72

Reguera, X. y Gutiérrez-Sánchez, Á. (2015). Implementación de un programa de Gimnasia Acrobática en Educación Secundaria para la mejora del autoconcepto. RETOS. Nuevas Tendencias en Educación Física, Deporte y Recreación, (27), 114117. Recuperado de http://www.redalyc.org/articulo.oa?id=345738764021

Redondo-Corcobado, P. y Fuentes, J. L. (2020). La investigación sobre el AprendizajeServicio en la producción científica española: una revisión sistemática. Revista Complutense de Educación, 31(1), 69-82. http://doi.org/10.5209/rced.61836

Ríos, M. (2008). La Educación Física en la Educación Social: una experiencia de aprendizaje y servicio. En M. Martínez (Ed.), Aprendizaje servicio y responsabilidad social de las universidades (pp. 113-128). Barcelona: Octaedro.

Schalock, R. L. y Verdugo, M. A. (2007). El concepto de calidad de vida en los servicios y apoyos para personas con DI. Revista Siglo Cero, 38(4), 21-36. Recuperado de https://sid.usal.es/10366/8-2-6

Sanz, I., Calle, M. T., Aguado, R. y Garoz, I. (2019). Adquisición de competencias para prevenir el accidente acuático: un proyecto ApS con estudiantes universitarios y población con diversidad funcional. Publicaciones, 49(4), 219-239. https:// doi.org/10.30827/publicaciones.v49i4.11737

Tapia, M. N. (2008). Aprendizaje y servicio solidario en la misión de la Educación Superior. En A. González y R. Montes (Eds.), Aprendizaje-servicio en la educación superior: una mirada analítica desde los protagonistas (pp. 11-33). Buenos Aires: Ministerio de Educación Presidencia de la Nación. Recuperado de http://www. bnm.me.gov.ar/giga1/documentos/EL001802.pdf

Vázquez-Toledo, S., Liesa-Orús, M. y Lozano-Roy, A. (2017). Recreos Cooperativos e Inclusivos a través de la metodología de Aprendizaje Servicio. Revista Electrónica Interuniversitaria de Formación del Profesorado, 20(1), 173-185. https://doi. org/10.6018/reifop/20.1.213181

Vernetta, M., López, J. L. y Panadero, F. (1996). El acrosport en la escuela. Barcelona: Inde. 